
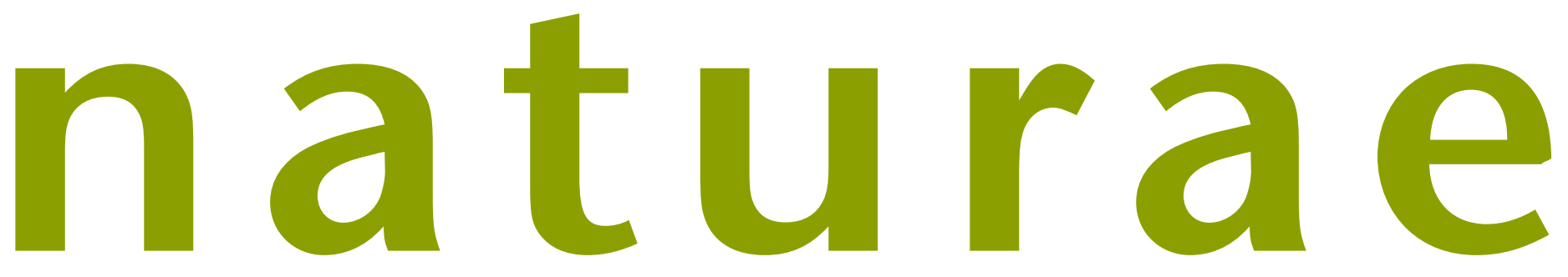

$2021 \cdot 5$
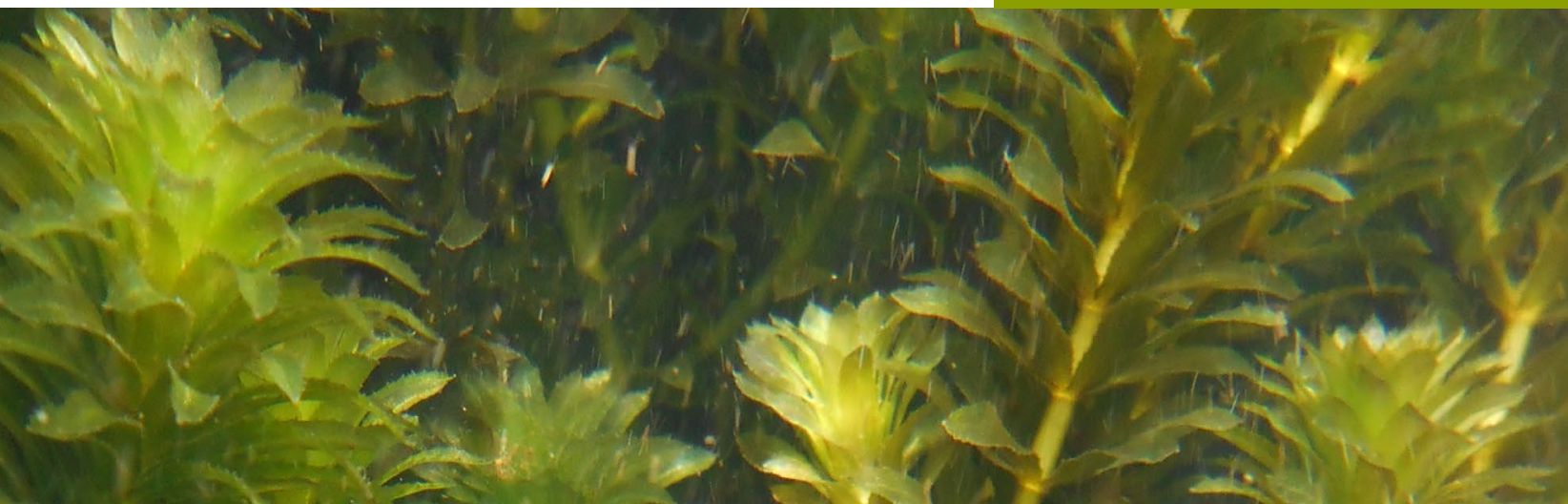

\title{
Détection precoce et lutte rapide contre la plante aquatique exotique Hydrilla verticillata (L.f.) Royle en Martinique
}

César DELNATTE, Cindy, TEPIÉ POTIRON \& Fabian RATEAU

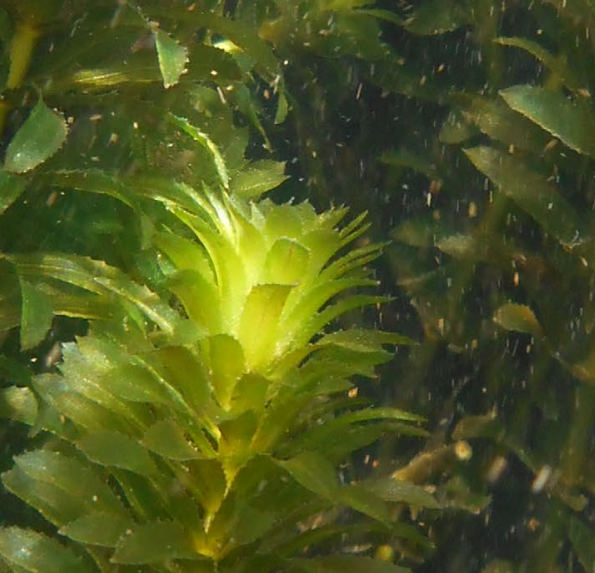


DiRECTEUR DE LA PUBLICATION / PUBLICATION DIRECTOR: Bruno David,

Président du Muséum national d'Histoire naturelle

RÉDACTEUR EN CHEF / EDITOR-IN-CHIEF: Jean-Philippe Siblet

ASSISTANTE DE RÉDACTION / ASSISTANT EDITOR: Sarah Figuet (naturae@mnhn.fr)

Mise EN PAGE / PAGE LAYOUT: Sarah Figuet

COMITÉ SCIENTIFIQUE / SCIENTIFIC BOARD:

Luc Abbadie (UPMC, Paris)

Luc Barbier (Parc naturel régional des caps et marais d'Opale, Colembert)

Aurélien Besnard (CEFE, Montpellier)

Vincent Boullet (Expert indépendant flore/végétation, Frugières-le-Pin)

Hervé Brustel (École d'ingénieurs de Purpan, Toulouse)

Patrick De Wever (MNHN, Paris)

Thierry Dutoit (UMR CNRS IMBE, Avignon)

Éric Feunteun (MNHN, Dinard)

Romain Garrouste (MNHN, Paris)

Grégoire Gautier (DRAAF Occitanie, Toulouse)

Olivier Gilg (Réserves naturelles de France, Dijon)

Frédéric Gosselin (Irstea, Nogent-sur-Vernisson)

Patrick Haffner (UMS PatriNat, Paris)

Frédéric Hendoux (MNHN, Paris)

Xavier Houard (OPIE, Guyancourt)

Isabelle Le Viol (MNHN, Concarneau)

Francis Meunier (Conservatoire d'espaces naturels - Hauts-de-France, Amiens)

Serge Muller (MNHN, Paris)

Francis Olivereau (DREAL Centre, Orléans)

Laurent Poncet (UMS PatriNat, Paris)

Nicolas Poulet (OFB, Vincennes)

Jean-Philippe Siblet (UMS PatriNat, Paris)

Laurent Tillon (ONF, Paris)

Julien Touroult (UMS PatriNat, Paris)

COUVERTURE / COVER:

Hydrilla verticillata (L.f.) Royle photographiée dans Rivière Case-Navire en novembre 2014. Crédit photo: C. Delnatte (DEAL Martinique).

Naturae est une revue en flux continu publiée par les Publications scientifiques du Muséum, Paris

Naturae is a fast track journal published by the Museum Science Press, Paris

Les Publications scientifiques du Muséum publient aussi / The Museum Science Press also publish:

Adansonia, Zoosystema, Anthropozoologica, European Journal of Taxonomy, Geodiversitas, Cryptogamie sous-sections Algologie, Bryologie, Mycologie, Comptes Rendus Palevol.

Diffusion - Publications scientifiques Muséum national d'Histoire naturelle

CP $41-57$ rue Cuvier F-75231 Paris cedex 05 (France)

Tél.: 33 (0)1 40794805 / Fax: 33 (0)1 40793840

diff.pub@mnhn.fr / http://sciencepress.mnhn.fr

(C) Publications scientifiques du Muséum national d'Histoire naturelle, Paris, 2021

ISSN (électronique / electronic): 1638-9387 


\title{
Détection précoce et lutte rapide contre la plante aquatique exotique Hydrilla verticillata (L.f.) Royle en Martinique
}

\author{
César DELNATTE \\ Office national des Forêts, \\ 78 route de Moutte, bp 578, F-97207 Fort-de-France cedex (Martinique) \\ cesar-augusto.delnatte@onf.fr \\ Cindy TEPIE POTIRON \\ Communauté d'Agglomération du Pays Nord Martinique, \\ 39 la Mairie, F-97225 Le Marigot (Martinique) \\ cindy.tepie@capnordmartinique.fr \\ Fabian RATEAU
}

Office national des Forêts international, 78 route de Moutte, bp 578, F-97207 Fort-de-France cedex (Martinique)

fabian.rateau@onf.fr

Soumis le 17 avril 2019 | Accepté le 5 octobre 2020 | Publié le 24 février 2021

MOTS CLÉS

Espèce exotique envahissante, lutte mécanique, Antilles françaises.
Delnatte C., Tepie Potiron C. \& Rateau F. 2021. - Détection précoce et lutte rapide contre la plante aquatique exotique Hydrilla verticillata (L.f.) Royle en Martinique. Naturae 2021 (5): 57-64. https://doi.org/10.5852/naturae2021a5

\section{RÉSUMÉ}

La problématique des espèces exotiques envahissantes est de plus en plus prise en compte au niveau de la Martinique qui semblait jusqu'alors présenter une certaine résistance par rapport à d'autres territoires insulaires tropicaux français. Ainsi, comme près de 70 autres espèces végétales exotiques préoccupantes et potentiellement envahissantes, les populations d'Hydrille verticillée (Hydrilla verticillata (L.f.) Royle) sont suivies à l'aide d'outils cartographiques par l'Office national des Forêts, la direction de l'environnement, de l'aménagement et du logement (DEAL) et le Conservatoire botanique de Martinique. Une campagne de lutte contre cette espèce a été menée en 2016 à la suite de nouvelles données de répartition remontées par le réseau de surveillance en place. Sur cette île des Antilles françaises, la combinaison de reliefs abrupts, issus d'épisodes volcaniques récents, et des régimes pluviométriques essentiellement corrélés aux précipitations, induit un régime torrentiel des cours d'eau. Nous émettons l'hypothèse que ce régime provoque la fragmentation des populations d'hydrophytes, ce qui favoriserait leur multiplication végétative. Ainsi, dans le cadre d'une détection rapide en vue d'une lutte précoce, la campagne d'éradication contre ces végétaux doit être entreprise avant la saison des pluies. De plus, la campagne menée confirme la difficulté de réguler l'espèce sur un sol à texture argileuse. Enfin, comme déjà énoncé dans le diagnostic datant de 2011, il est nécessaire de mettre en place un comité local traitant spécifiquement des espèces exotiques envahissantes afin de prioriser et coordonner les actions.

\section{ABSTRACT}

Early detection and control of exotic aquatic plant $\mathrm{Hydrilla} \mathrm{verticillata} \mathrm{(L.f.)} \mathrm{Royle} \mathrm{in} \mathrm{Martinique} \mathrm{(West} \mathrm{Indies).}$ The problem of invasive alien species is increasingly being taken into consideration in Martinique, which seemed to have a certain resilience compared to other French tropical island territories. In this way, like nearly 70 alien and potentially invasive plant species, populations of Verticillate hydrilla (Hydrilla verticillata (L.f.) Royle) are monitored using mapping tools by the National Forestry Office, the Government Agency in charge of Environment and the Botanical Conservatory of Martinique. 
KEY WORDS

Invasive alien species, mechanical control, French West Indies.
Following new distribution data reported by the existing monitoring network, a control campaign was conducted in 2016. In this island of French West Indies, the combination of steep reliefs, resulting from recent volcanic episodes, and rainfall patterns, induce a torrential regime of streams. We hypothesize that this torrential regime would cause the fragmentation of submersed macrophyte populations which would encourage their vegetative multiplication. Thus, in the context of early detection and rapid response, the eradication campaign against these plants must occur before the rainy season. Moreover, this campaign confirms the difficulty of regulating the species in a clay soil. Finally, as already stated in 2013 , it is necessary to set up a local committee dealing specifically with invasive alien species in order to prioritize and coordinate actions.

\section{INTRODUCTION}

Depuis 2005 (Millenium Ecosystem Assessment 2005), les invasions biologiques sont reconnues comme étant la deuxième cause de perte de biodiversité à l'échelle mondiale dans les îles tropicales après la destruction des habitats. Les espèces exotiques envahissantes (EEE) engendrent des impacts directs et indirects affectant les habitats, les services écosystémiques, les activités économiques, la santé humaine et induisent une homogénéisation biotique. Celle-ci est définie comme le remplacement des communautés végétales indigènes par un faible nombre d'espèces exotiques qui peuvent coexister avec les activités humaines (McKinney \& Lockwood 2001). Cette mutation, induisant la régression ou la disparition des espèces autochtones, nous laisse entrevoir les notions de Nouvelle Pangée et d'Homogocène avec une biocénose exotique, cosmopolite et peu diversifiée. Comme le prophétisait déjà Elton (1958), si on se projette suffisamment loin, l'état final du monde biologique sera plus simple et plus pauvre.

De par leur isolement évolutif, leur faible superficie, leurs taux d'endémisme et leurs déséquilibres taxonomiques et fonctionnels, les milieux insulaires sont particulièrement vulnérables aux invasions biologiques (Loope \& Mueller-Dombois 1989; Denslow 2003). De plus, les îles de la ceinture tropicale rencontrent également une occurrence plus élevée de catastrophes naturelles telles que les cyclones, glissements de terrains, éruptions volcaniques et séismes. Ceux-ci induisent des perturbations favorisant les invasions biologiques (Hobbs \& Huenneke 1992; Bhattarai \& Cronin 2014).

L'antenne caribéenne du Centre international pour l'Agriculture et la Bioscience (CABI) a réalisé en 2003 une synthèse comptabilisant 552 espèces introduites dont 390 naturalisées ou envahissantes à l'échelle de l'arc caribéen (Kairo et al. 2003). Malgré l'énorme travail de synthèse réalisé, force est de constater que les données sont lacunaires. En effet, pour la Martinique où, sur les 3200 espèces végétales vasculaires recensées, plus de la moitié de la flore est d'origine allochtone (DEAL Martinique comm. pers.), les chiffres de 2003 comptabilisaient 37 espèces introduites dont sept naturalisées ou envahissantes. À ce jour et depuis 2014, plus de 70 espèces végétales introduites par l'homme, et envahissantes et préoccupantes, sont suivies à l'aide d'outils cartographiques (Annexe 1).
La présence en Martinique de l'Hydrille verticillée (Hydrilla verticillata (L. F.) Royle) est connue depuis 2005 et peu après, Maddi \& Brizard (2010) alertaient déjà sur son fort potentiel d'envahissement. C'est pourquoi, quand en 2016, l'Office de l'Eau (ODE) a identifié de nouvelles stations avec de faibles populations, il nous est paru opportun de rapidement mener une campagne pour tenter d'éliminer l'espèce sur la rivière Bois d'Inde.

La DEAL de Martinique, en partenariat avec l'ONF, l'Office de l'Eau et le Conservatoire botanique de Martinique, concentre les informations dans des bases de données, avec géolocalisation, de plus de 70 espèces végétales préoccupantes. De plus, dans le cadre de la transcription en droit français du règlement européen (UE nº 1143/2014) relatifà la prévention et à la gestion de l'introduction et de la propagation des EEE, elle met à disposition des listes d'espèces animales et végétales envahissantes régulièrement mises à jour sur son site Internet. Ces données sont transmises aux partenaires qui en font une demande justifiée.

\section{LA MARTINIQUe ET SES MILIEUX AQUATIQUeS}

Île de la Caraïbe, d'une superficie de $1128 \mathrm{~km}^{2}$, la Martinique se situe en position centrale dans l'arc des Petites Antilles entre la Dominique au nord et Sainte-Lucie au sud. Elle est baignée par l'océan Atlantique à l'est et par la mer des Caraïbes à l'ouest.

La Caraïbe fait partie d'un des 36 points chauds de la biodiversité mondiale (Myers et al. 2000; Williams et al. 2011; Noss et al. 2015). Cette classification met en évidence les zones ayant une forte biodiversité avec au moins 1500 espèces de plantes vasculaires endémiques mais subissant également des menaces avec une perte de $70 \%$ des habitats naturels originels (Myers et al. 2000). De plus, en se basant sur l'endémisme et leur ratio ramené à la superficie, les îles de la Carä̈be font partie, selon Shi et al. (2005), d'un des six points les plus chauds («hottest hotspots») de biodiversité.

Avec 41 taxa vasculaires endémiques, la Martinique est l'île la plus riche à l'échelle des Petites Antilles en nombre d'espèces endémiques rapportées à la surface. De plus, recensant plus de 400 espèces d'arbre, l'île héberge $87 \%$ de la flore arborée ligneuse de cet archipel qui en compte 461 espèces (Fiard comm. pers.).

Le climat y est de type tropical maritime chaud, avec $26^{\circ} \mathrm{C}$ de température moyenne annuelle et humide avec une hygrométrie de $80 \%$ en mars-avril et de $87 \%$ en octobre-novembre (Agence d'urbanisme et d'aménagement de la Martinique 2015). 
On distingue deux saisons, «le carême», période chaude et relativement sèche de janvier à avril et "l'hivernage», saison des pluies de juillet à octobre, qui se caractérise également par un risque cyclonique important. Ces deux périodes sont entrecoupées par des intersaisons de transition.

L'effet orographique joue un très grand rôle dans la répartition de la pluviométrie. En effet, la pluviosité présente également des variations entre les versants atlantiques, au vent, et caraïbes, sous le vent.

Sur l'île, les unités du relief s'organisent en massifs. Au nord, le paysage est marqué par la Montagne Pelée culminant à 1397 m et les Pitons du Carbet, dont le Piton Lacroix atteint $1196 \mathrm{~m}$. Dans la partie centrale, la plaine du Lamentin est bordée par des collines localement appelés «mornes» et dont l'altitude varie de 300 à $350 \mathrm{~m}$. À l'est, la montagne du Vauclin, avec ses $504 \mathrm{~m}$, jalonne le paysage. Au sud, le morne Larcher $(477 \mathrm{~m})$ et le morne Bigot $(460 \mathrm{~m})$ caractérisent le relief collinéen de la presqu'île des Anses d'Arlet.

On dénombre 161 rivières en Martinique (Agence d'urbanisme et d'aménagement de la Martinique 2015) dont près de trente sont pérennes (DIREN \& ODE 2010), ce qui représente environ $1000 \mathrm{~km}$ d'écoulements permanents (Marras 2014). Parce que la majorité des bassins versants sont de petite dimension, que les réserves souterraines en eau sont de faible importance comparées à l'écoulement de surface, les débits des cours d'eau ont des variations saisonnières corrélées aux précipitations. Ainsi, l'écoulement est maximal en juillet-août et septembre (Guiscafre et al. 1976). Toutefois, il n'est pas rare de rencontrer tout au long de l'année des crues importantes y compris pendant la période sèche du carême (Marras 2014).

Au nord de l'île, les cours d'eau ont les caractéristiques des «rivières de montagne" avec des talwegs (zones basses d'une vallée ou du lit d'un cours d'eau, principalement modelées par l'érosion) encaissés, de fortes pentes et des bassins allongés. Au sud, elles sont de type "rivières de plaine et de mangrove" avec des bassins versants moins allongés et des vallées plus larges (SDAGE de la Martinique 2001).

Les reliefs ajoutés aux régimes pluviométriques induisent le caractère torrentiel de la plupart des rivières de l'île (Guiscafre et al. 1976). Ainsi, les variations importantes des débits, associées aux crues, induisent une déstructuration fréquente des lits des cours d'eau par un arrachement et un charriage des matériaux. Ce transport solide, maximal sur le nord, est estimé à plusieurs milliers de mètres cubes de matériaux charriés à chaque crue importante (SDAGE de la Martinique 2001), hors phénomènes exceptionnels de type lahars (coulées boueuses de débris rocheux des volcans).

\section{HYDRILLA VERTICILLATA (L. F.) ROYLE}

L'Hydrille verticillée est une espèce de la famille des Hydrocharitacées. C'est une hydrophyte submergée dont les tiges peuvent dépasser deux mètres de long et s'enraciner jusqu'à $30 \mathrm{~cm}$ de profondeur. Elle se développe dans des eaux douces avec une profondeur pouvant atteindre $15 \mathrm{~m}$ (Maddi \& Brizzard 2010). Elle produit de nombreux stolons et rhizomes et près de la surface ses ramifications deviennent plus denses. Sa croissance est optimale pour des températures comprises

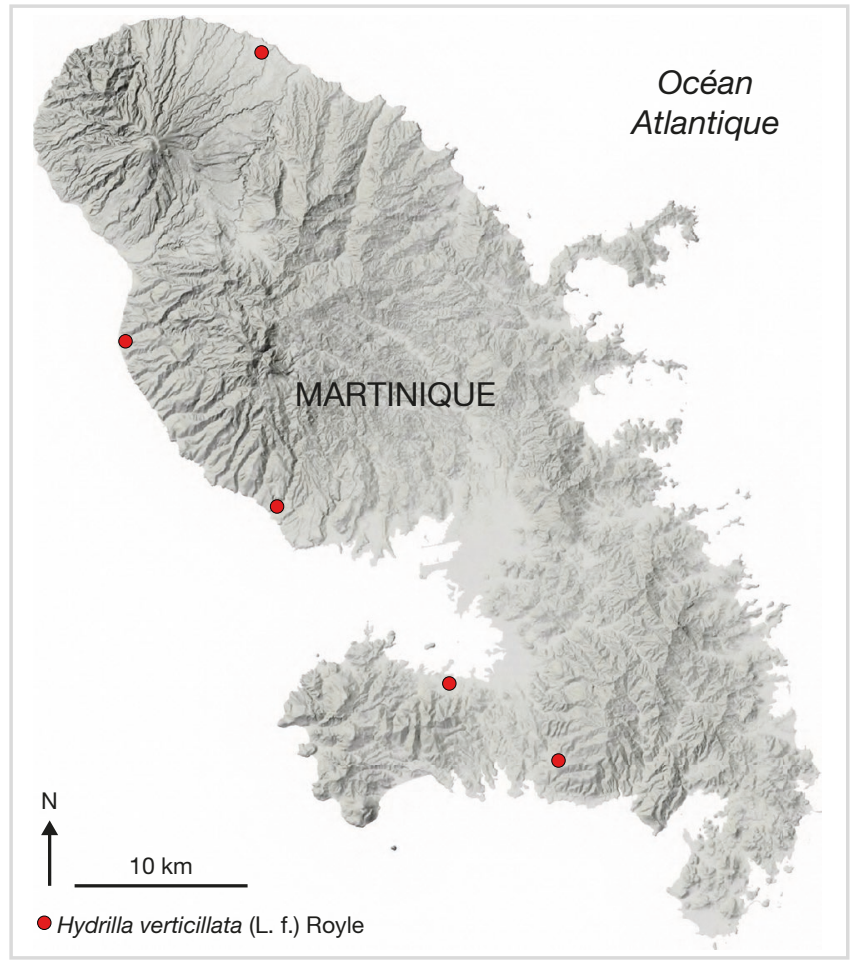

FIG. 1. - Carte de répartition d'Hydrilla verticillata (L. F.) Royle, 1839 en Martinique en 2017. Source: Relief de la Martinique 2016 - GéoMartinique

entre 20 et $27^{\circ} \mathrm{C}$. Bien qu'elle préfere les hydrosystèmes lentiques, elle peut se développer dans des eaux vives, pourvu que son système racinaire s’y soit ancré (Langeland 1996).

L'espèce se multiplie préférentiellement par voie végétative, soit par bouturage, soit par propagation de tubercules enterrés, soit par turions. Ces derniers sont produits sur les axes foliaires ainsi qu'à l'extrémité de ses rhizomes (Langeland 1996).

Elle a actuellement une répartition cosmopolite (https://www. gbif.org/species/5329570, dernière consultation le 1er octobre 2018), dont l'origine asiatique est généralement admise (Maddi \& Brizzard 2010). Sa première signalisation en Floride date de 1960 et en Dominique, île voisine située au nord de la Martinique, elle remonte à 1983 (Maddi \& Brizzard 2010). Bien que déjà observée en 2005, c'est en 2010 que cette information remonte aux services de l'État en Martinique. Deux stations sont alors recensées, l'une à Basse-Pointe, au nord de l'île et l'autre aux Trois Îlets dans le sud-ouest (Maddi \& Brizzard 2010). En 2016, l'Office de l'Eau (ODE) a découvert de nouvelles stations sur les communes de Scholcher (Rivière Case Navire), du Carbet (Rivière du Carbet) et de Sainte-Luce (Rivière Bois d'Inde) (Fig. 1).

Dans les collectivités d'outre-mer, l'espèce a également été signalée en Guadeloupe et en Nouvelle-Calédonie. Par ailleurs, à La Réunion, cette espèce est considérée comme indigène et a été évaluée comme étant en danger (EN) (https://mascarine. cbnm.org, dernière consultation le $1^{\text {er }}$ octobre 2018).

\section{Impact sur les écosystèmes}

Les impacts de l'Hydrille verticillée sur les écosystèmes aquatiques sont bien connus (Langeland 1996; http://vecteurs.cqeee. org/wp-content/uploads/sites/5/2014/04/CQEEE_Hydrille_ver- 
ticillee.pdf; https://www.cabi.org/isc/datasheet/28170, dernière consultation le $1^{\text {er }}$ octobre 2018). Espèce exotique envahissante, elle remplace les communautés végétales autochtones et modifie les habitats. L'espèce est un compétiteur pour les ressources, notamment la lumière pour les couches inférieures et les gaz dissous. De par le volume qu'elle peut occuper, elle réduit le débit des cours d'eau, bouche les installations hydroélectriques et les systèmes d'irrigation. Elle peut provoquer des embâcles et ainsi induire des inondations. Elle perturbe également la navigation et la pêche. Enfin, l'espèce favorise les gîtes de ponte des moustiques, vecteurs de maladies infectieuses (chikungunya, dengue, paludisme, zika, etc.).

Au vu de l'ensemble de ces impacts, l'espèce a également des conséquences économiques avec des frais induits par les dégâts engendrés, les activités qu'elle perturbe ainsi que les moyens de lutte engagés.

\section{Détection précoce et lutte rapide}

Bien que l'on sache que de nombreuses espèces introduites observent une phase de latence avant une rapide extension, trop souvent, une espèce exotique n'est considérée comme envahissante qu'à partir de l'observation de couverts denses monospécifiques, que ses populations sont visibles et que les impacts sur les biocénoses autochtones sont perceptibles (Soubeyran 2008).

En 2008, la neuvième conférence des Parties de la Convention sur la Diversité biologique, dans sa décision IX/4, invitait les pays signataires à collaborer au développement et à l'utilisation des mécanismes de réponse rapide. Plus récemment, le règlement $\left(n^{\circ} 1143 / 2014\right)$ du Parlement européen et du Conseil du 22 octobre 2014 relatif à la prévention et à la gestion de l'introduction et de la propagation des espèces exotiques envahissantes précise qu'il est essentiel de mettre en œuvre des mesures d'éradication rapide afin d'empêcher l'établissement et la propagation des EEE.

La lutte rapide se définit comme le fait de maitriser ou éradiquer une espèce exotique envahissante alors que sa population et ses impacts sont encore limités et localisés (UICN France 2015).

Selon Simberloff et al. (2013) la détection précoce et la lutte rapide constituent la seconde ligne de défense après la prévention. La détection précoce et la lutte rapide permettent de limiter les coûts humains et financiers tout en augmentant les chances de réussite de limiter l'expansion voire d'éradiquer les EEE concernées. En cas de réussite, on prévient alors les coûts économiques de la lutte sur le long terme (UICN France 2015). Enfin, selon Loope et al. (2006), cette méthode se révèle être actuellement la solution la plus efficace pour lutter contre les invasions biologiques.

\section{MATÉRIEL \& MÉTHODE}

Dans le lit de la rivière Bois d'Inde, située au quartier Volcart sur la commune de Sainte-Luce, trois peuplements monospécifiques ont été observés le 21 juin 2016 (données en WGS84 UTM20N : 720989-1602708 puis 720976-1602708 et 720972-1602709).
Ce site a été choisi pour mener une lutte car l'espèce y avait été détectée récemment et que les populations étaient suffisamment réduites pour procéder à un arrachage manuel (Fig. 2).

Contrairement aux méthodes mécaniques et chimiques qui peuvent modifier totalement le biotope et les biocénoses, celleci permet une régulation de l'espèce en limitant, de manière localisée, les impacts sur le milieu naturel.

\section{DESCRIPTION DE LA MÉTHODE UTILISÉE}

La première étape consiste en la mise en tension d'un filet à mailles de dimension $2 \mathrm{~mm}$ sur $4 \mathrm{~mm}$, en travers du cours d'eau et en aval du chantier. Une partie du filet doit être immergée de façon à retenir les tubercules et turions qui s'échapperaient durant l'arrachage.

Ensuite, la progression de l'arrachage doit s'effectuer de l'aval vers l'amont pour éviter que des matériaux en suspension n'opacifient l'eau. Il convient de s'attarder sur le système racinaire en évitant que la racine ne se casse. Les plants arrachés sont récupérés dans les sacs.

Il est important que l'exportation de ces plants soit faite hors du lit majeur afin d'éviter qu'ils ne se retrouvent à nouveau dans la rivière à cause du ruissellement et des crues.

En fin de manipulation, on procède au rangement et au nettoyage du filet. Ce dernier est replié sur lui-même afin de retenir les éléments capturés. Les éventuels boutures, turions et tubercules récoltés dans la toile doivent également être exportés en dehors du lit majeur.

La première campagne d'arrachage a été effectuée le 5 juillet 2016. Par manque de temps, l'espèce n'a pas pu être complètement éradiquée. En deux heures, environ $20 \mathrm{~m}^{2}$ ont été nettoyés. Le $1^{\text {er }}$ août, une visite de contrôle a permis d'observer une forte régression des peuplements sans propagation en aval ainsi qu'une bonne décomposition des plantes exportées hors du lit de la rivière.

Une seconde campagne d'arrachage a été effectuée, en période d'étiage, le 7 février 2017. Le volume à exporter hors du cours d'eau était alors bien moindre. En effet, en 45 minutes, une superficie avoisinant les $15 \mathrm{~m}^{2}$ a pu être nettoyée, celle-ci correspond à la totalité du peuplement alors observé.

\section{DISCUSSION}

Le 4 avril 2017, une prospection fait état de nombreuses petites populations fragmentées s'étalant depuis le site initial jusqu'à près de 150 mètres en aval. Les interventions des mois de juillet et de février n'ont donc pas permis la suppression des populations d'Hydrille verticillée.

Cette expérimentation vient confirmer les observations de Langeland (1996) qui stipule que l'efficacité de la lutte mécanique varie en fonction des saisons, en fonction de la fréquence des interventions, et qu'elle est inefficace dans les zones où l'espèce est ancrée dans un sol argileux.

On peut émettre l'hypothèse que le charriage des matériaux induit par les régimes torrentiels, caractéristique de la majorité des cours d'eau martiniquais en période de crue, provoque une importante fragmentation des populations. Il apparaitt 


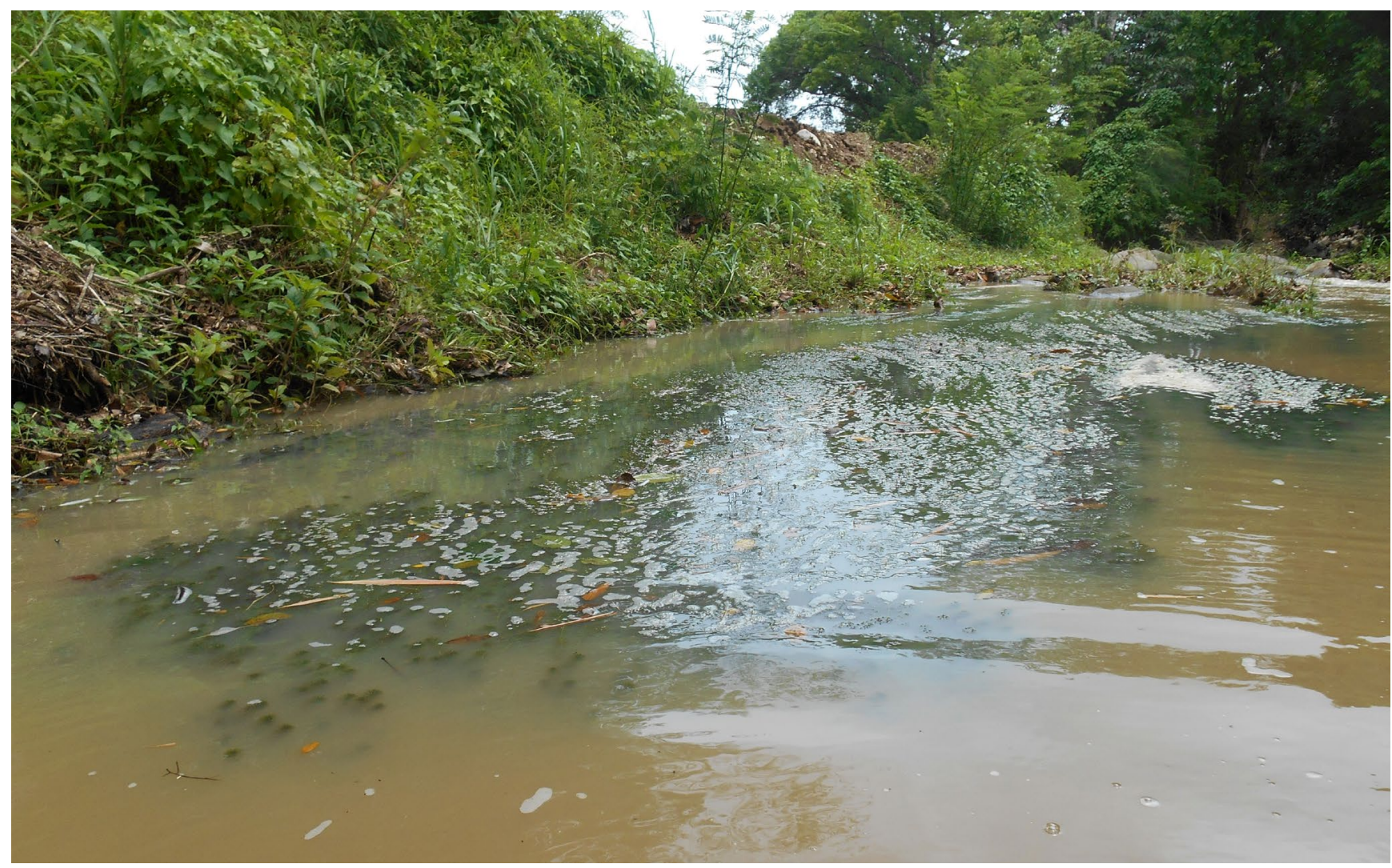

FIG. 2. - Peuplement d'Hydrilla verticillata (L.f.) Royle observé le 21 juin 2016. Crédit photo: C. Delnatte.

donc que la lutte mécanique des populations d'hydrophytes qui se multiplient facilement par voie végétative doit se terminer avant l'arrivée de la saison des pluies.

La méthode d'arrachage est très chronophage dans un sol argileux. En effet, il nécessite un travail minutieux pour ne pas libérer des boutures et des turions, ni laisser des fragments de tubercules dans le sol. Par ailleurs, en fonction de la turbidité de l'eau qui dépend de la saison, il est difficile d'estimer le travail effectué et de récupérer tout le système racinaire. De plus, il plus facile pour les boutures, turions et tubercules d'échapper à la vigilance.

La station observée dans l'estuaire de la rivière du Carbet a aujourd'hui disparu. On peut émettre une autre hypothèse, à savoir que les incursions marines issues des grandes marées ou celles liées aux phénomènes cycloniques modifient la salinité sur une période assez longue pour éliminer l'espèce.

La Martinique semblait jusqu'à présent présenter une certaine résistance aux espèces exotiques envahissantes (Joseph 2007), cependant la problématique prend de l'ampleur avec une amélioration des connaissances et une intensification récente des prospections botaniques.

Historiquement, dans l'île, pour les végétaux, l'ONF était la seule autre structure ayant mené des actions contre des espèces exotiques envahissantes, en l'occurrence le Tulipier du Gabon (Spathodea campanulata P. Beauv.) et la Petite citronnelle (Triphasia trifolia (Burm.f.) P. Wilson) courant 2013.
Depuis 2014, le «réseau sentinelle" regroupe l'ODE, l'ONF et le Parc naturel de Martinique (PNM). Dans ce cadre, les agents de ces trois structures ont une mission de surveillance et d'alerte des pressions dans les bassins versants et les cours d'eau en plus des pollutions, prélèvements, travaux en rivière, etc. Les EEE aquatiques constituent une de leurs compétences. À ce jour, c'est le seul réseau de surveillance traitant des invasions biologiques mis en place localement. Il serait intéressant de développer ce réseau à d'autres habitats que ceux dulçaquicoles, mais aussi d'ouvrir ce réseau à l'Observatoire martiniquais de la Biodiversité (OMB), plateforme locale du Système d'Information sur la Nature et les Paysages (SINP) qui enregistre et cartographie les inventaires et observations naturalistes. De plus d'autres structures, acteurs de terrain, comme le dispositif de surveillance biologique du territoire (Direction de l'Agroalimentaire, de l'Agriculture et de la Forêt, Chambre d'Agriculture, Fédération régionale de Défense contre les Organismes nuisibles) et les brigades de l'environnement des communautés de communes, ont des compétences permettant d'étendre ce réseau.

En effet, comme souligné dans le diagnostic et état des lieux des invasions biologiques aux Antilles françaises (Asconits Consultants et al. 2011), il manque en Martinique un comité local ou un groupe de travail pour coordonner les moyens, hiérarchiser et organiser les actions mais avant tout, rédiger un document de référence présentant une stratégie globale. 
Notons que depuis le début de la rédaction de ce manuscrit, la DEAL, l'ONF et le Conservatoire botanique de Martinique ont hiérarchisé, avec l'échelle d'invasibilité de Lavergne, plus de 130 espèces végétales. L'arrêté du 9 août 2019 relatif à la prévention de l'introduction et de la propagation des espèces végétales exotiques envahissantes sur le territoire de la Martinique a été publié. De plus, l'initiative sur les espèces exotiques envahissantes en outre-mer du comité français de l'UICN a organisé en février 2020 un atelier sur la problématique en Martinique.

\section{Remerciements}

Les auteurs remercient Séverine François, Séverine Laporte et Jean-Yves Meyer pour leur relecture attentive et leurs conseils avisés, ainsi que Vanessa Corre et Pascal Maras pour leurs prêts d'ouvrages traitant d'hydrologie et de livres rares. Les auteurs remercient également Clara Zimmer pour la réalisation de la carte ainsi que les deux rapporteurs, Serge Muller et Fabien Barthelat, qui ont grandement contribué à l'amélioration de l'article.

\section{RÉFÉRENCES}

Agence d'Urbanisme et d'Aménagement de la Martinique 2015. - L'eau en Martinique. CAUE Martinique, Fort-deFrance, 242 p. (Coll. Regards croisés)

Asconit Consultants, Pareto \& Impact Mer 2011. - Diagnostic sur l'invasion biologique aux Antilles françaises. Stratégie de suivi et de prévention. Phase 1: État des lieux des connaissances. DEAL, Basse Terre, Fort-de-France, 143 p.

BhatTARAi G. P. \& CrOnin J. T. 2014. - Hurricane activity and the large-scale pattern of spread of an invasive plant species. Plos ONE 9 (5): e98478. https://doi.org/10.1371/journal.pone.0098478

DENSLOW J. S. 2003. - Weeds in paradise: thoughts on the invasibility of tropical islands. Annals of the Missouri Botanical Garden 90 (1): 229-127. https://doi.org/10.2307/329831

DIREN \& ODE 2010. - Qualité des milieux aquatiques de la Martinique de 1999 à 2008. DIREN/ODE, Fort-de-France, 74 p.

ELTON C. S. 1958. - The Ecology of Invasions by Animals and Plants. University of Chicago Press, Chicago, $196 \mathrm{p}$.

Guiscafre J., Klein J.-C. \& MONIOD F. 1976. — Les Ressources en eau de surface de la Martinique. Office de la Recherche scientifique et technique Outre-Mer (coll. Monographies hydrologiques; 4), Paris, 180 p.

HobBs R. J. \& HuENNEKE L. F. 1992. — Disturbance, diversity, and invasion: implications for conservation. Conservation Biology 6 (3): 324-337. https://doi.org/10.1046/j.1523-1739.1992.06030324.x

JosepH P. 2007. — La question des epèces végétales invasives dans les Petites Antilles. Le cas de l'île de la Martinique, in DAVID G. (éd.), Espaces tropicaux et risque, du local au global. IRD; Presses universitaires, Orléans: 328-339.

Kairo M., Ali B., Cheesman O., Haysom K. \& Murphy S. 2003. - Invasive Species Threats in the Caribbean Region. Report to the Nature Conservancy. CAB International, Egham, 134 p.
LANGELAND K. A. 1996. - Hydrilla verticillata (L.f.) Royle (Hydrocharitaceae), "The perfect aquatic weed». Castanea 61: 293-304

Loope L. L. \& MuelLer-Dombois D. 1989. - Characteristics of invaded islands, with special references to Hawaii, in DRAKE J. A., Mooney F., Di Castri F., Groves R. H., Kruger F. J., RejMANEK M. \& Williamson M. (éds), Biological Invasions, a Global Perspective. John Wiley \& Sons Ltd, Chichester: 525 p.

Loope L., Sheppard A., Pascal M. \& Jourdan H. 2006. L'éradication: une mesure de gestion des populations allochtones, in Beauvais M.-L., Coleno A., Jourdan H, Chouchan D. (éds), Espèces envahissantes dans l'archipel néo-calédonien. IRD, Paris: 396-412.

MADDI F. A. \& BRIZARD J.-P. 2010. - Hydrilla verticillata (L.f.) Royle, une nouvelle espèce envahissante en Martinique? Situation actuelle et prospective. Bulletin de la Société naturelle de l'Ouest de la France nouvelle série, 32 (1): 16-31.

MARRAS P. 2014. - L’hydrométrie en Martinique. Mémoire présenté pour l'obtention du titre d'ingénieur diplômé par l'État spécialité eau et environnement. DEAL Martinique, Schoelcher; Polytech, Montpellier, $79 \mathrm{p}$.

McKinney M. L. \& Lockwood J. L. 2001. — Biotoc Homogenization: A Sequential and Selective Process, in Lockwood J. \& McKinney M. (éds), Biotic Homogenization. Kluwer Academic/ Plenum Publishers, New York: 1-17.

MilleNium ECOSYSTEM ASSESSMENT 2005. - Current state \& trends, volume 1. Findings of the condition and trends working group. Island press, Washington, Covelo, London, 596 p.

Myers N., Mittermeier R. A., MitTermeier C. G., Da FonSECA G. A. B. \& KENT J. 2000. - Biodiversity hotspots for conservation priorities. Nature 403: 853-858. https://doi. org/10.1038/35002501

Noss R. F., Platt W. J., Sorrie B. A., Weakley A. S., Means D. B., Costanza J. \& PeEt R. K. 2015. - How global biodiversity hotspots may go unrecognized: lessons from the North American Coastal Plain. Diversity and Distributions 21: 236-344. https:// doi.org/10.1111/ddi.12278

SDAGE DE LA MarTiniQue 2001. - Volume 2, État des lieuxDiagnostic. SDAGE Martinique, Fort-de-France, 76 p.

Shi H., Singh A., Kant S., ZhU Z. \& Waller E. 2005. - Integrating habitats status, human population pressure, and protection status into biodiversity conservation priority setting. Conservation Biology 19 (4): 1272-1285. https://doi.org/10.1111/j.15231739.2005.00225.x

Simberloff D., Martin J.-L., Genovesi P., Maris V., Wardle D. A., Aronson J., Courchamp F., Galil B., Garcia-Berthou E., Pascal M., Pysek P., Sousa R., Tabacchi E. \& Vila M. 2013. - Impacts of biological invasions: what's what and the way forward. Trends in Ecology and Evolution 28 (1): 58-66. https://doi.org/10.1016/j.tree.2012.07.013

SoubEYRAN Y. 2008. - Espèces exotiques envahissantes dans les collectivités françaises d'outre-mer. État des lieux et recommandations. Comité français de l'UICN (coll. Planète Nature), Paris, 202 p.

UICN FRANCE 2015. - Guide pratique pour la détection précoce et la réaction rapide face aux espèces exotiques envahissantes dans les collectivités françaises d'outre-mer. Principes généraux, lignes directrices et options de mise en cuvre. UICN, Paris, $74 \mathrm{p}$.

Williams K. J., Ford A., Rosauer D., De Silva N., Mittermeier R., Bruce C., Larsen F.W. \& Margules C. 2011. - Forests of East Australia: the 35th biodiversity hotspot, in ZACHOS F. E. \& HABEL J. C. (éds), Biodiversity hotspots: distribution and protection of conservation priority areas. Springer, Heidelberg: 295-310. 
ANNEXE

ANNEXE 1. - Liste des espèces végétales introduites suivies en Martinique.

\begin{tabular}{|c|c|}
\hline Famille & Nom scientifique \\
\hline Acanthaceae & $\begin{array}{l}\text { Asystasia gangetica (L.) T.Anderson } \\
\text { Odontonema tubaeforme (Bertol.) Kuntze } \\
\text { Sanchezia speciosa Leonard } \\
\text { Thunbergia alata Bojer ex Sims } \\
\text { Thunbergia grandiflora (Roxb. ex Rottl.) Roxb. }\end{array}$ \\
\hline Apocynaceae & $\begin{array}{l}\text { Calotropis procera (Aiton) W.T Aiton } \\
\text { Cryptostegia madagascariensis Bojer ex Decne. } \\
\text { Funtumia elastica (P.Preuss) Stapf }\end{array}$ \\
\hline Araceae & $\begin{array}{l}\text { Epipremnum aureum (Linden \& André) G.S Bunting } \\
\text { Syngonium podophyllum Schott } \\
\text { Pistia stratiotes L. }\end{array}$ \\
\hline Asparagaceae & $\begin{array}{l}\text { Sansevieria trifasciata Prain } \\
\text { Sansevieria hyacinthoides (L.) Druce }\end{array}$ \\
\hline Asteraceae & $\begin{array}{l}\text { Erigeron karvinskianus DC. } \\
\text { Xanthium strumarium L. } \\
\text { Tithonia diversifolia (HemsI.) A.Gray } \\
\text { Erigeron bellioides DC. }\end{array}$ \\
\hline $\begin{array}{l}\text { Bignoniaceae } \\
\text { Casuarinaceae }\end{array}$ & $\begin{array}{l}\text { Spathodea campanulata P.Beauv. } \\
\text { Casuarina equisetifolia L. }\end{array}$ \\
\hline $\begin{array}{l}\text { Combretaceae } \\
\text { Commelinaceae }\end{array}$ & $\begin{array}{l}\text { Terminalia catappa L. } \\
\text { Callisia fragrans (Lindl.) Woodson } \\
\text { Tradescantia zebrina Heynh. ex Bosse } \\
\text { Tradescantia spathacea Sw. }\end{array}$ \\
\hline $\begin{array}{l}\text { Crassulaceae } \\
\text { Cucurbitaceae }\end{array}$ & $\begin{array}{l}\text { Kalanchoe pinnata (Lam.) Pers. } \\
\text { Coccinia grandis (L.) Voigt }\end{array}$ \\
\hline $\begin{array}{l}\text { Cyperaceae } \\
\text { Euphorbiaceae }\end{array}$ & $\begin{array}{l}\text { Cyperus papyrus L. } \\
\text { Clerodendrum quadriloculare (Blanco) Merr. }\end{array}$ \\
\hline Fabaceae & $\begin{array}{l}\text { Jacaranda mimosifolia D.Don } \\
\text { Acacia auriculiformis A.Cunn. ex Benth. } \\
\text { Adenanthera pavonina L. } \\
\text { Albizia lebbeck (L.) Benth. } \\
\text { Mimosa arenosa (Willd.) Poir. } \\
\text { Dichrostachys cinerea (L.) Wight \& Arn. } \\
\text { Flemingia strobilifera (L.) W.T.Aiton }\end{array}$ \\
\hline Goodeniaceae & Scaevola taccada (Gaertn.) Roxb. \\
\hline $\begin{array}{l}\text { Hydrocharitaceae } \\
\text { Lamiaceae }\end{array}$ & $\begin{array}{l}\text { Hydrilla verticillata (L.f.) Royle } \\
\text { Gmelina arborea Roxb. } \\
\text { Clerodendrum chinense (Osbeck) Mabb. } \\
\text { Clerodendrum paniculatum L. }\end{array}$ \\
\hline Lauraceae & $\begin{array}{l}\text { Cinnamomum verum J.Presl } \\
\text { Litsea glutinosa (Lour.) C.B.Rob. }\end{array}$ \\
\hline Limnocharitaceae & Limnocharis flava (L.) Buchenau \\
\hline $\begin{array}{l}\text { Malvaceae } \\
\text { Marattiaceae }\end{array}$ & $\begin{array}{l}\text { Heliocarpus donnellsmithii Rose ex J.D. Smith } \\
\text { Angiopteris evecta (G.Forst.) Hoffm. }\end{array}$ \\
\hline Melastomataceae & $\begin{array}{l}\text { Heterotis rotundifolia (Sm.) Jacq.-Fél. } \\
\text { Arthrostemma ciliatum Pav.ex D.Don } \\
\text { Miconia calvescens DC. }\end{array}$ \\
\hline Meliaceae & Melia azedarach L. \\
\hline $\begin{array}{l}\text { Moraceae } \\
\text { Myrtaceae }\end{array}$ & $\begin{array}{l}\text { Castilla elastica Sessé } \\
\text { Psidium cattleanium Sabine } \\
\text { Syzygium jambos (L.) Alston }\end{array}$ \\
\hline $\begin{array}{l}\text { Nephrolepidaceae } \\
\text { Orchidaceae }\end{array}$ & $\begin{array}{l}\text { Nephrolepis brownii (Desv.) Hoverkamp \& Miyam } \\
\text { Dendrobium crumenatum Sw. } \\
\text { Oeceoclades maculata (Lindl.) Lindl. } \\
\text { Spathoglottis plicata Blume }\end{array}$ \\
\hline $\begin{array}{l}\text { Pinaceae } \\
\text { Poaceae }\end{array}$ & $\begin{array}{l}\text { Pinus caribaea Morelet } \\
\text { Cenchrus purpureus (Schumach.) Morrone } \\
\text { Cenchrus setaceus (Forssk.) Morrone } \\
\text { Melinis minutiflora P.Beauv. } \\
\text { Urochloa maxima (Jacq.) R.D.Webster } \\
\text { Bambusa vulgaris Schrad. ex J.C.Wendl. }\end{array}$ \\
\hline $\begin{array}{l}\text { Polygonaceae } \\
\text { Pontederiaceae }\end{array}$ & $\begin{array}{l}\text { Antigonon leptopus Hook. \& Arn. } \\
\text { Eichhornia crassipes (Mart.) Solms }\end{array}$ \\
\hline
\end{tabular}


ANNEXE 1. - Suite.

\section{Famille}

Rubiaceae

Rutaceae

Salviniaceae

Selaginellaceae

Sphenocleaceae

Thelypteridaceae

Zingiberaceae

\section{Nom scientifique}

\section{Morinda citrifolia L.}

Triphasia trifolia (Burm.f.) P.Wilson

Salvinia molesta D.S.Mitch.

Selaginella willdenowii (Desv. ex Poir.) Baker

Sphenoclea zeylanica Gaertn.

Macrothelypteris torresiana (Gaudich.) Ching

Hedychium gardnerianum Sheppard ex Ker Gawl.

Hedychium coronarium J.Koenig 